PROCEEDINGS OF THE

AMERICAN MATHEMATICAL SOCIETY

Volume 139, Number 6, June 2011, Pages 1897-1907

S 0002-9939(2011)10895-5

Article electronically published on January 21, 2011

\title{
THE LINEAR DUAL OF THE DERIVED CATEGORY OF A SCHEME
}

\author{
CARLOS SANCHO DE SALAS AND FERNANDO SANCHO DE SALAS
}

(Communicated by Lev Borisov)

\begin{abstract}
Let $X \rightarrow S$ be a projective morphism of schemes. We study the category $D(X / S)^{*}$ of $S$-linear exact functors $D(X) \rightarrow D(S)$, and we study the Fourier transform $D(X) \rightarrow D(X / S)^{*}$.
\end{abstract}

\section{INTRODUCTION}

Let $p: X \rightarrow S$ be an $S$-scheme. Let $D(X)=D_{q c}(X)$ be the derived category of complexes of $\mathcal{O}_{X}$-modules with quasi-coherent cohomology. Given an object $K \in D(X)$, we define the functor

$$
\begin{aligned}
\omega_{K}: D(X) & \rightarrow D(S) \\
M & \mapsto p_{*}(K \otimes M) .
\end{aligned}
$$

(Since we are dealing with derived categories all functors are assumed to be derived and we use the abbreviated notation: $p_{*}=\mathbf{R} p_{*}, \otimes=\stackrel{\mathrm{L}}{\otimes}$, etc.). This functor satisfies the following properties:

1) It is an additive, graded (i.e., commutes with translations) and is an exact functor (i.e., takes exact triangles into exact triangles).

2) It is $S$-linear: One has a natural isomorphism $\omega_{K}\left(M \otimes p^{*} N\right) \simeq \omega_{K}(M) \otimes N$, for any $M \in D(X), N \in D(S)$. This follows by the projection formula.

An $S$-linear form on $D(X)$ is a functor $\omega: D(X) \rightarrow D(S)$ satisfying 1) and endowed with an isomorphism such as 2 ). An $S$-linear morphism between $S$-linear forms is defined in the obvious way. We shall denote by $D(X / S)^{*}$ the category of $S$-linear forms and $S$-linear morphisms and call it the $S$-linear dual category of $D(X)$. An $S$-linear form of the type $\omega_{K}, K \in D(X)$, is called an integral linear form (of kernel $K$ ). We have a functor (which we call a Fourier transform)

$$
\text { Fourier: } \begin{aligned}
D(X) & \rightarrow D(X / S)^{*} \\
K & \mapsto \omega_{K} .
\end{aligned}
$$

An $S$-linear form $\omega: D(X) \rightarrow D(S)$ is said to be bounded and coherent (resp. perfect) if it maps $D_{c}^{b}(X)$ into $D_{c}^{b}(S)$ (resp. $D_{\text {perf }}(X)$ into $D_{\text {perf }}(S)$ ). We shall denote by $D(X / S)_{b c}^{*}\left(\right.$ resp. $\left.D(X / S)_{\text {perf }}^{*}\right)$ the faithful subcategory of $D(X / S)^{*}$ whose

Received by the editors January 27, 2010.

2010 Mathematics Subject Classification. Primary 14F05; Secondary 18E30.

Key words and phrases. Derived categories, integral functors, linear functors.

This work was supported by research projects MTM2009-07289 (MEC) and SA001A07 (JCYL).

(C)2011 American Mathematical Society Reverts to public domain 28 years from publication 
objects are the bounded and coherent $S$-linear forms (resp. the perfect $S$-linear forms).

Assume now for simplicity that $S$ is regular and $X \rightarrow S$ is projective. The main result of the paper is to prove that the Fourier transform induces equivalences

$$
D_{c}^{b}(X) \stackrel{\sim}{\longrightarrow} D(X / S)_{\text {perf }}^{*}, \quad D_{\text {perf }}(X) \stackrel{\sim}{\longrightarrow} D(X / S)_{b c}^{*} .
$$

In other words, every perfect $S$-linear form (resp. bounded and coherent $S$-linear form) is integral and its kernel is a unique bounded and coherent (resp. perfect) object of $D(X)$. This is obtained in Theorem 1.14 and Corollary 1.16, where $S$ is not assumed to be regular. Indeed, the only hypothesis in Theorem 1.14 (resp. Corollary 1.16) is the projectivity of the morphism $X \rightarrow S$ (resp. projectivity and finite Tor-dimension). Even flatness is not required. Hence these results apply for very general situations. We have also proved these results for the $S$-linear dual categories of $D_{c}^{b}(X)$ and $D_{\text {perf }}(X)$. See Theorems 1.23 and 1.26

These results are connected with Orlov's result about the integrality of exact functors. Orlov proved in 6 that if $X$ and $Y$ are smooth projective varieties over a field $k$ and $F: D_{c}^{b}(X) \rightarrow D_{c}^{b}(Y)$ is exact and fully faithful, then $F$ is an integral functor; i.e., there exists an object $K \in D_{c}^{b}(X \times Y)$ such that $F \simeq \Phi_{K}$, where $\Phi_{K}$ is the integral functor with kernel $K$ :

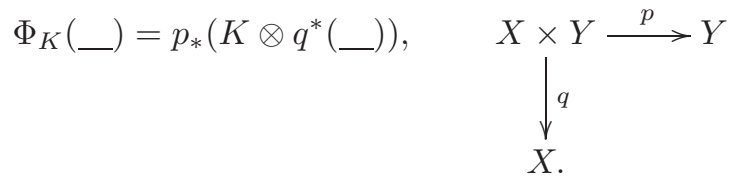

This has been generalized to singular schemes in 7 and 2]. However, there are many integral functors that are not fully faithful. It is widely believed that this result should hold also without the faithfulness hypothesis: is any exact functor integral? One could go further and state the integrality conjecture in a relative setting: let $X \rightarrow S$ and $Y \rightarrow S$ be two projective $S$-schemes (and assume for simplicity that all the schemes are regular) and let $K$ be an object in $D_{c}^{b}\left(X \times_{S} Y\right)$. One has a (relative) integral functor

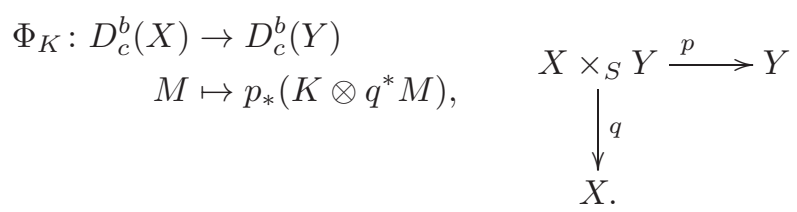

This functor is exact and $S$-linear. One could ask if any exact $S$-linear functor from $D_{c}^{b}(X)$ to $D_{c}^{b}(Y)$ is of this type. It is not difficult to find a counterexample if we do not assume $Y \rightarrow S$ to be flat. Our result proves the conjecture in the case $Y=S$. But in this case our result is not only an integrality result because it is also shown that the morphisms between the kernels are the same as the $S$-linear morphisms between the associated functors, which is known to fail in general (i.e. for $Y \neq S$ ).

Regarding the general integrality conjecture, under the flatness assumption on $Y \rightarrow S$, we give a linear criterion for the integrality of a functor in Proposition 1.30.

Moreover, our results give new evidence of the validity of the integrality conjecture. If $F: D_{c}^{b}(X) \rightarrow D_{c}^{b}(Y)$ is a (relative) integral functor with kernel $K \in$ $D_{c}^{b}\left(X \times{ }_{S} Y\right)$, then this same kernel defines a functor in the opposite direction. That 
is, a kernel $K$ has two associated functors $F: D_{c}^{b}(X) \rightarrow D_{c}^{b}(Y)$ and $F^{*}: D_{c}^{b}(Y) \rightarrow$ $D_{c}^{b}(X)$, with the same kernel. Let us say that these are partners. Our results prove that any exact $S$-linear functor $F: D_{c}^{b}(X) \rightarrow D_{c}^{b}(Y)$ has a partner $F^{*}: D_{c}^{b}(Y) \rightarrow$ $D_{c}^{b}(X)$, which is nothing but the "dual" functor.

\section{LineAR FUnCTORS}

We shall denote $D(X)=D_{q c}(X)$ to be the derived category of complexes of $\mathcal{O}_{X}$-modules with quasi-coherent cohomology. A functor $F: D(X) \rightarrow D(Y)$ means an additive, graded and exact functor, i.e., an additive functor that commutes with the shift functor and takes exact triangles into exact triangles. For simplicity all schemes are assumed to be quasi-compact, quasi-separated and of finite type over a field $k$. Whenever one has a morphism of schemes $f: X \rightarrow Y$, we still denote by $f$ the morphism $X \times_{Y} Y^{\prime} \rightarrow Y^{\prime}$ induced after a base change $Y^{\prime} \rightarrow Y$.

Definition 1.1. Let $p: X \rightarrow S$ and $q: Y \rightarrow S$ be two $S$-schemes. An $S$-linear functor $F: D(X) \rightarrow D(Y)$ is a covariant functor endowed with a bi-additive and bi-graded bi-functorial isomorphism $\theta_{F}(M, E): F\left(M \otimes p^{*} E\right) \simeq F(M) \otimes q^{*} E$. That is, an $S$-linear functor is a pair $\left(F, \theta_{F}\right)$, though we shall usually denote it by $F$.

An $S$-linear morphism $\phi: F \rightarrow F^{\prime}$ of $S$-linear functors is a morphism of functors which is compatible with the $\theta$ 's, i.e., such that the diagram

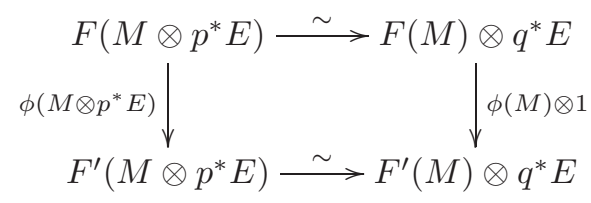

is commutative.

Definition 1.2. Let $X$ be an $S$-scheme. An $S$-linear form on $D(X)$ is an $S$-linear functor $\omega: D(X) \rightarrow D(S)$. A morphism of $S$-linear forms is an $S$-linear morphism of functors.

We shall denote by $D(X / S)^{*}$ the category of $S$-linear forms on $D(X)$ and $S$ linear morphisms.

Example. Let $K \in D\left(X \times{ }_{S} Y\right)$. Let us denote by $p: X \times{ }_{S} Y \rightarrow Y$ and $q: X \times{ }_{S} Y \rightarrow$ $X$ the natural projections. The functor $\Phi_{K}: D(X) \rightarrow D(Y)$ defined by

$$
\Phi_{K}(M)=p_{*}\left(q^{*} M \otimes K\right)
$$

is $S$-linear, with the $\theta$ provided by the projection formula. We say that $\Phi_{K}$ is a relative integral functor of kernel $K$. In particular, for each $K \in D(X)$, we have an $S$-linear form on $D(X)$ :

$$
\omega_{K}: D(X) \rightarrow D(S), \quad M \mapsto p_{*}(M \otimes K) .
$$

We say that $\omega_{K}$ is an integral $S$-linear form on $D(X)$ of kernel $K$.

Definition 1.3. We say that a functor $F: D(X) \rightarrow D(Y)$ is bounded and coherent if it takes $D_{c}^{b}(X)$ into $D_{c}^{b}(Y)$. We say that $F$ is perfect if it takes $D_{\text {perf }}(X)$ into $D_{\text {perf }}(Y)$. We say that $F$ is quasi-perfect if it takes $D_{\text {perf }}(X)$ into $D_{c}^{b}(Y)$.

Definition 1.4. A morphism $f: X \rightarrow Y$ is of finite Tor-dimension if $f^{*}: D(Y) \rightarrow$ $D(X)$ is bounded and coherent. 


\subsection{Complexes of finite homological dimension over $S$.}

Definition 1.5. Let $p: X \rightarrow S$ be an $S$-scheme. An object $M \in D(X)$ is said to be of finite homological dimension over $S$ (fhd over $S$ for short) if the functor

$$
\begin{aligned}
D(S) & \rightarrow D(X) \\
E & \mapsto p^{*} E \otimes M
\end{aligned}
$$

is bounded and coherent. We shall denote by $D_{\text {fhd } / S}(X)$ the faithful subcategory of $D(X)$ whose objects are the complexes of finite homological dimension over $S$.

Remark 1.6. (1) If $S$ is a regular scheme, then $D_{\text {fhd } / S}(X)=D_{c}^{b}(X)$.

(2) If $f$ has finite Tor-dimension (for example $f$ flat or $S$ regular), then $D_{\text {perf }}(X)$ $\subset D_{\text {fhd } / S}(X)$.

(3) If $f$ has finite Tor-dimension and $X$ is regular, then $D_{\text {perf }}(X)=D_{\text {fhd } / S}(X)$ $=D_{c}^{b}(X)$.

(4) If $X=S$, then $D_{\text {fhd } / S}(S)=D_{\text {perf }}(S)$.

We shall mention some properties of complexes of finite homological dimension over $S$. They can be found in [4].

Proposition 1.7. Assume that $p: X \rightarrow S$ is projective and let $\mathcal{O}_{X}(1)$ be a relatively ample invertible sheaf on $X$. Let $M \in D(X)$. The following conditions are equivalent:

(1) $M$ is fhd over $S$.

(2) $p_{*}\left(M \otimes \mathcal{O}_{X}(n)\right)$ is perfect for any $n$.

(3) The functor $\mathbf{R} \mathcal{H o m}_{X}^{\bullet}\left(M, p^{!}(\ldots)\right): D(S) \rightarrow D(X)$ is bounded coherent.

Two important properties of complexes of finite homological dimension over $S$ are the following:

Proposition 1.8. Assume that $p: X \rightarrow S$ is locally projective. Let us denote by $D_{X / S}=p^{!} \mathcal{O}_{S}$ the relative dualizing complex and for any $M \in D(X)$ let us denote by $M^{\vee}=\mathbf{R} \mathcal{H o m}_{X}^{\bullet}\left(M, D_{X / S}\right)$. If $K \in D_{\text {fhd } / S}(X)$, then

(1) For any $N \in D(S)$ one has an isomorphism

$$
K^{\vee} \otimes p^{*} N \stackrel{\sim}{\rightarrow} \mathbf{R H o m}_{X}^{\bullet}\left(K, p^{!} N\right) .
$$

(2) $K^{\vee}$ is of finite homological dimension over $S$ and the natural map $K \rightarrow K^{\vee \vee}$ is an isomorphism.

Definition 1.9. We say that a functor $F: D(X) \rightarrow D(Y)$ is of finite homological dimension over $S$ (fhd over $S$ for short) if it takes $D_{\text {fhd } / S}(X)$ into $D_{\text {fhd } / S}(Y)$. We say that $F$ is quasi-fhd over $S$ if it takes $D_{\text {fhd } / S}(X)$ into $D_{c}^{b}(Y)$.

Proposition 1.10. Assume that $p: X \rightarrow S$ is projective and let $\omega_{K}: D(X) \rightarrow$ $D(S)$ be an integral $S$-linear form. Then

(1) $\omega_{K}$ is bounded and coherent $\Leftrightarrow K$ is perfect.

(2) $\omega_{K}$ is perfect $\Leftrightarrow K$ is fhd over $S$.

(3) $\omega_{K}$ is quasi-perfect $\Leftrightarrow K$ is bounded and coherent.

Proof. (1) Assume that $\omega_{K}$ is bounded and coherent. Let us see that $K$ is perfect. It suffices to see that $K \otimes N$ is bounded and coherent for any bounded and coherent $N$. For this, it suffices to see that $p_{*}\left(K \otimes N \otimes \mathcal{O}_{X}(n)\right)$ is bounded and coherent for 
any $n$ (see [4, Lemma 2.5] and its following paragraph). Since $p_{*}\left(K \otimes N \otimes \mathcal{O}_{X}(n)\right)=$ $\omega_{K}\left(N \otimes \mathcal{O}_{X}(n)\right)$, one concludes the proof of part (1). The converse is clear.

(2) Assume that $\omega_{K}$ is perfect. Then $\omega_{K}\left(\mathcal{O}_{X}(n)\right)$ is perfect for any $n$; i.e., $p_{*}\left(K \otimes \mathcal{O}_{X}(n)\right)$ is perfect and then $K$ is fhd over $S$ by Proposition 1.7. Conversely, assume that $K$ is fhd over $S$ and let $M \in D_{\text {perf }}(X)$. Then, for any $N \in D_{c}^{b}(S)$, $\omega_{K}(M) \otimes N=\omega_{K}\left(M \otimes p^{*} N\right)=p_{*}\left(K \otimes M \otimes p^{*} N\right)$ is bounded and coherent. Hence $\omega_{K}(M)$ is perfect.

(3) Assume that $\omega_{K}$ is quasi-perfect. Then $p_{*}\left(K \otimes \mathcal{O}_{X}(n)\right)=\omega_{K}\left(\mathcal{O}_{X}(n)\right)$ is bounded and coherent; hence $K$ is bounded and coherent. The converse is clear.

Proposition 1.11. Let $F: D(X) \rightarrow D(Y)$ be an $S$-linear functor. If $F$ is bounded and coherent, then it is fhd over $S$.

Proof. Let $P \in D_{\text {fhd } / S}(X)$. For any $N \in D_{c}^{b}(S), P \otimes p^{*} N$ is bounded and coherent and then $F\left(P \otimes p^{*} N\right)$ is bounded and coherent. Since $F$ is $S$-linear, it follows that $F(P) \otimes q^{*} N$ is bounded and coherent. Hence $F(P)$ is fhd over $S$.

Proposition 1.12. Assume that $p: X \rightarrow S$ is of finite Tor-dimension and let $\omega: D(X) \rightarrow D(S)$ be an $S$-linear form on $D(X)$. Then

$\omega$ is fhd over $S$ (resp. quasi-fhd over $S$ ) $\Rightarrow \omega$ is perfect (resp. quasi-perfect).

Proof. Since $p$ is of finite Tor-dimension, $D_{\text {perf }}(X) \subset D_{\text {fhd } / S}(X)$. One concludes the proof because $D_{\text {fhd } / S}(S)=D_{\text {perf }}(S)$.

Notation. Let $p: X \rightarrow S$ be an $S$-scheme. We shall denote by $D(X / S)_{\text {perf }}^{*}$ (resp. $\left.D(X / S)_{\text {bc }}^{*}, D(X / S)_{\text {fhd } / S}^{*}, D(X / S)_{\text {quasi-perf }}^{*}, D(X / S)_{\text {quasi-fhd } / S}^{*}\right)$ the category of perfect (resp. bounded and coherent, fhd $/ S$, quasi-perfect, quasi fhd $/ S$ ) $S$-linear forms.

Proposition 1.13. Let $p: X \rightarrow S$ be a projective morphism. If $\omega: D(X) \rightarrow D(S)$ is an $S$-linear form, then it has a right adjoint.

Proof. Brown representability (see [5]) says that $\omega$ has a right adjoint if we assume that $\omega$ commutes with infinite direct sums. But one can copy the proof, replacing this condition by the $S$-linearity one.

Theorem 1.14. Let $p: X \rightarrow S$ be a projective morphism. The functor

$$
\begin{aligned}
D_{f h d / S}(X) & \rightarrow D(X / S)_{\mathrm{perf}}^{*} \\
K & \mapsto \omega_{K}
\end{aligned}
$$

is an equivalence.

Proof. Sketch: We shall construct a quasi-inverse $D(X / S)_{\text {perf }}^{*} \rightarrow D_{\text {fhd } / S}(X)$ in the following way: let $\omega^{\#}: D(S) \rightarrow D(X)$ be the right adjoint of $\omega$, and define $K_{\omega}=\mathbf{R} \mathcal{H o m}{ }_{X}\left(\omega^{\#}\left(\mathcal{O}_{S}\right), D_{X / S}\right)$, where $D_{X / S}$ is the relative dualizing complex of $X$ over $S$. We shall see that the correspondences $K \mapsto \omega_{K}$ and $\omega \mapsto K_{\omega}$ give the desired equivalence.

Now we give more details. By Proposition 1.13, $\omega$ has a right adjoint $\omega^{\#}: D(S) \rightarrow$ $D(X)$. So one has

$$
\operatorname{Hom}_{D(S)}(\omega(M), N) \simeq \operatorname{Hom}_{D(X)}\left(M, \omega^{\#}(N)\right) .
$$

Let us see that this yields an isomorphism

$$
\mathbf{R} \mathcal{H o m} \dot{S}_{S}(\omega(M), N) \stackrel{\sim}{\longrightarrow} p_{*} \mathbf{R} \mathcal{H o m} \cdot \dot{X}\left(M, \omega^{\#}(N)\right) .
$$


In fact, for any $E \in D(S)$ one has

$$
\begin{aligned}
\operatorname{Hom}_{D(S)}\left(E, \mathbf{R H o m}_{S}(\omega(M), N)\right) & \simeq \operatorname{Hom}_{D(S)}(E \otimes \omega(M), N) \\
& \simeq \operatorname{Hom}_{D(S)}\left(\omega\left(p^{*} E \otimes M\right), N\right) \\
& \simeq \operatorname{Hom}_{D(X)}\left(p^{*} E \otimes M, \omega^{\#}(N)\right) \\
& \simeq \operatorname{Hom}_{D(S)}\left(E, p_{*} \mathbf{R} \mathcal{H o m} \dot{x}_{X}\left(M, \omega^{\#}(N)\right)\right) .
\end{aligned}
$$

If one takes $M=\mathcal{O}_{X}(-n)$ one obtains

$$
\mathbf{R} \mathcal{H o m}_{S}^{\bullet}\left(\omega\left(\mathcal{O}_{X}(-n)\right), N\right) \simeq p_{*}\left(\omega^{\#}(N) \otimes \mathcal{O}_{X}(n)\right) .
$$

Assume that $\omega$ is perfect. Then, if $N$ is perfect, $\omega^{\#}(N)$ is of finite homological dimension over $S$ : in fact, if $N$ is perfect, then $\mathbf{R} \mathcal{H} \operatorname{lom}_{S}\left(\omega\left(\mathcal{O}_{X}(-n)\right), N\right)$ is perfect, because $\omega\left(\mathcal{O}_{X}(-n)\right)$ is perfect. Then $p_{*}\left(\omega^{\#}(N) \otimes \mathcal{O}_{X}(n)\right)$ is perfect for any $n$ by (1.1). By Proposition 1.7 $\omega^{\#}(N)$ is fhd over $S$.

Let us denote by $D_{X / S}=f^{!} \mathcal{O}_{S}$ the relative dualizing complex and let $M^{\vee}=$ $\mathbf{R} \mathcal{H o m}_{X}^{\bullet}\left(M, D_{X / S}\right)$. Let us denote

$$
K_{\omega}=\omega^{\#}\left(\mathcal{O}_{S}\right)^{\vee} .
$$

By Proposition 1.8, $K_{\omega}$ has finite homological dimension over $S$ and $\omega^{\#}\left(\mathcal{O}_{S}\right) \stackrel{\sim}{\longrightarrow}$ $K_{\omega}^{\vee}$. Let us prove that

$$
\mathbf{R} \mathcal{H o m}_{X}^{\bullet}\left(K_{\omega}, p^{!} N\right) \simeq \omega^{\#}(N) .
$$

Let us first define a morphism $\mathbf{R H o m}_{X}^{\bullet}\left(K_{\omega}, p^{!} N\right) \rightarrow \omega^{\#}(N)$. Since $K_{\omega}$ has finite homological dimension over $S$ one has $\omega^{\#}\left(\mathcal{O}_{S}\right) \otimes p^{*} N \simeq \mathbf{R} \mathcal{H} o m_{X}^{\bullet}\left(K_{\omega}, p^{!} N\right)$. So one has to define a morphism $\omega^{\#}\left(\mathcal{O}_{S}\right) \otimes p^{*} N \rightarrow \omega^{\#}(N)$. The unit morphism $\left(\omega \circ \omega^{\#}\right)\left(\mathcal{O}_{S}\right) \rightarrow \mathcal{O}_{S}$ induces a morphism $\left(\omega \circ \omega^{\#}\right)\left(\mathcal{O}_{S}\right) \otimes N \rightarrow N$. By the $S$ linearity of $\omega$ one has a morphism $\omega\left(\omega^{\#}\left(\mathcal{O}_{S}\right) \otimes p^{*} N\right) \rightarrow N$ and then a morphism $\omega^{\#}\left(\mathcal{O}_{S}\right) \otimes p^{*} N \rightarrow \omega^{\#}(N)$. Now let us see that it is an isomorphism. It suffices to see that it is an isomorphism after tensoring by $\mathcal{O}_{X}(n)$ and taking the direct image $p_{*}$. But

$$
\begin{aligned}
p_{*}\left(\omega^{\#}\left(\mathcal{O}_{S}\right) \otimes p^{*} N \otimes \mathcal{O}_{X}(n)\right) & \simeq p_{*}\left(\omega^{\#}\left(\mathcal{O}_{S}\right) \otimes \mathcal{O}_{X}(n)\right) \otimes N \\
& \stackrel{\text { 1.1.1 }}{\simeq} \mathbf{R H o m} \cdot\left(\omega\left(\mathcal{O}_{X}(-n)\right), \mathcal{O}_{X}\right) \otimes N \\
& \simeq \mathbf{R} \mathcal{H o m}_{S}\left(\omega\left(\mathcal{O}_{X}(-n)\right), N\right) \\
& \stackrel{\text { 1.1. }}{\simeq} p_{*}\left(\omega^{\#}(N) \otimes \mathcal{O}_{X}(n)\right) .
\end{aligned}
$$

We have then proved that $\omega^{\#} \simeq \mathbf{R} \mathcal{H o m}_{X}^{\bullet}\left(K_{\omega}, p^{!}(\quad)\right)$. Taking left adjoints one concludes that $\omega \simeq \omega_{K_{\omega}}$. Moreover $K_{\omega}$ is fhd over $S$. The uniqueness of $K_{\omega}$ follows from the construction.

Conversely, if $K$ is fhd over $S$, then $\omega_{K}$ is perfect by Proposition 1.10 and $K_{\omega_{K}} \simeq K$. Indeed, the right adjoint of $\omega_{K}$ is

$$
\omega_{K}^{\#}\left(\_\right)=\mathbf{R} \mathcal{H o m} \cdot m_{S}\left(K, p^{!}\left(\_\right)\right) \simeq K^{\vee} \otimes p^{*}\left(\_\right)
$$

by Proposition 1.8 and then $K_{\omega_{K}}=\omega_{K}^{\#}\left(\mathcal{O}_{S}\right)^{\vee} \simeq K^{\vee \vee} \simeq K$ by (2) of Proposition 1.8 .

The correspondences $\omega \mapsto K_{\omega}$ and $K \mapsto \omega_{K}$ are clearly functorial. To conclude, we have to see that a morphism $K \rightarrow L$ in $D_{\text {fhd } / S}(X)$ is equivalent to an $S$ linear morphism of functors $\omega_{K} \rightarrow \omega_{L}$. Giving an $S$-linear morphism $\omega_{K} \rightarrow \omega_{L}$ is 
equivalent to giving an $S$-linear morphism $\omega_{L}^{\#} \rightarrow \omega_{K}^{\#}$. By (1.2), this is equivalent to giving a morphism $L^{\vee} \rightarrow K^{\vee}$. One concludes by (2) of Proposition 1.8 .

Remark 1.15. (1) In the proof of the theorem it has been shown that one can relax the hypothesis of $\omega$ being perfect and replace it by the weaker hypothesis: $\omega\left(\mathcal{O}_{X}(i)\right)$ is perfect for any $i$.

(2) The kernel $K_{\omega}$ associated to an $S$-linear form $\omega$ does not depend on the $S$-linear structure of $\omega$, since it was defined as $\omega^{\#}\left(\mathcal{O}_{S}\right)^{\vee}$, with $\omega^{\#}$ the right adjoint of $\omega$. The $S$-linear structure of $\omega$ is used to construct the isomorphism $\omega \simeq \omega_{K_{\omega}}$. One deduces that the $S$-linear structure of $\omega$ is essentially unique: assume that $(\omega, \theta)$ and $\left(\omega, \theta^{\prime}\right)$ are two $S$-linear structures on $\omega$. Since $K_{(\omega, \theta)}=K_{\left(\omega, \theta^{\prime}\right)}$, one obtains an $S$-linear isomorphism $(\omega, \theta) \simeq$ $\left(\omega, \theta^{\prime}\right)$.

Corollary 1.16. Let $p: X \rightarrow S$ be a projective morphism of finite Tor-dimension. The functor

$$
\begin{aligned}
D_{\text {perf }}(X) & \rightarrow D(X / S)_{b c}^{*} \\
K & \mapsto \omega_{K}
\end{aligned}
$$

is an equivalence.

Proof. Let $\omega$ be a bounded and coherent $S$-linear form on $D(X)$. By Proposition 1.11, $\omega$ is fhd over $S$, and hence it is perfect by Proposition 1.12. By Theorem 1.14, $\omega$ is isomorphic to $\omega_{K}$, with $K \in D_{\text {fhd } / S}(X)$. Finally $K$ is perfect by Proposition 1.10

Corollary 1.17. Let $p: X \rightarrow S$ be a projective morphism and assume that $S$ is regular. Then

$$
D(X / S)_{\text {quasi-perf }}^{*}=D(X / S)_{\text {perf }}^{*} \simeq D_{c}^{b}(X)=D_{\text {fhd } / S}(X)
$$

and

$$
D(X / S)_{\mathrm{fhd} / S}^{*}=D(X / S)_{\mathrm{bc}}^{*} \simeq D_{\mathrm{perf}}(X) .
$$

Proof. Since $S$ is regular, $D_{\text {perf }}(S)=D_{c}^{b}(S)$ and then $D(X / S)_{\text {quasi-perf }}^{*}=$ $D(X / S)_{\text {perf }}^{*} \quad$ By Theorem 1.14. $D(X / S)_{\text {perf }}^{*} \simeq D_{\text {fhd } / S}(X)$. Finally $D_{c}^{b}(X)=$ $D_{\text {fhd } / S}(X)$ because $S$ is regular.

For the second part, $D(X / S)_{\mathrm{fhd} / S}^{*}=D(X / S)_{\mathrm{bc}}^{*}$ because $D_{c}^{b}(X)=D_{\text {fhd } / S}(X)$ and $D_{c}^{b}(S)=D_{\text {fhd } / S}(S)$. Finally, $D(X / S)_{\mathrm{bc}}^{*} \simeq D_{\text {perf }}(X)$ by Corollary 1.16 .

Corollary 1.18. Let $p: X \rightarrow S$ be a projective morphism of finite Tor-dimension and assume that $X$ is regular. Then

$$
D_{c}^{b}(X)=D_{\text {perf }}(X)=D_{\text {fhd } / S}(X)
$$

and

$$
\begin{aligned}
D(X / S)_{\text {quasi-perf }}^{*}=D(X / S)_{\text {perf }}^{*} & =D(X / S)_{\mathrm{fhd} / S}^{*}=D(X / S)_{\mathrm{bc}}^{*} \\
& =D(X / S)_{\text {quasi-fhd } / S}^{*} \simeq D_{c}^{b}(X) .
\end{aligned}
$$

Proof. $D_{c}^{b}(X)=D_{\text {perf }}(X)$ because $X$ is regular, and $D_{c}^{b}(X)=D_{\text {fhd } / S}(X)$ because $f$ has finite Tor-dimension and $X$ is regular. It follows that $D(X / S)_{\mathrm{fhd} / S}^{*}=$ $D(X / S)_{\text {perf }}^{*}$ and $D(X / S)_{\mathrm{bc}}^{*}=D(X / S)_{\text {quasi-fhd } / S}^{*}=D(X / S)_{\text {quasi-perf. }}^{*}$ Now 
$D(X / S)_{\mathrm{perf}}^{*} \simeq D_{\mathrm{fhd} / S}(X)$ by Theorem 1.14, and $D(X / S)_{\mathrm{bc}}^{*} \simeq D_{\text {perf }}(X)$ by Corollary 1.16 .

As an immediate consequence of Theorem 1.14 and Corollary 1.16 one obtains the "partner" of a functor:

Corollary 1.19. Let $X$ and $Y$ be two projective $S$-schemes and let $F: D(X) \rightarrow$ $D(Y)$ be a perfect $S$-linear functor. Then it induces a functor:

$$
F^{*}: D_{\text {fhd } / S}(Y) \rightarrow D_{\text {fhd } / S}(X) .
$$

Corollary 1.20. Let $X$ and $Y$ be two projective $S$-schemes of finite Tor-dimension over $S$ and let $F: D(X) \rightarrow D(Y)$ be a bounded and coherent $S$-linear functor. Then it induces a functor:

$$
F^{*}: D_{\text {perf }}(Y) \rightarrow D_{\text {perf }}(X) .
$$

Of course, if $F$ is both perfect and bounded and coherent, the partners $F^{*}$ of Corollaries 1.19 and 1.20 coincide.

Remark 1.21. If $F: D(X) \rightarrow D(Y)$ is a (relative) integral functor of kernel $K \in$ $D\left(X \times{ }_{S} Y\right)$, then $F^{*}$ is also an integral functor with the same kernel.

1.2. Dual categories of $D_{\text {perf }}(X)$ and $D_{c}^{b}(X)$. In this subsection we shall reproduce the results of the previous one for the categories $D_{\text {perf }}(X)$ and $D_{c}^{b}(X)$. That is, we want to study the categories of $S$-linear functors $D_{\text {perf }}(X) \rightarrow D_{\text {perf }}(S)$ and $D_{c}^{b}(X) \rightarrow D_{c}^{b}(S)$.

We shall study first the linear dual of $D_{\text {perf }}(X)$.

Definition 1.22. Let $p: X \rightarrow S$ be an $S$-scheme. An $S$-linear form on $D_{\text {perf }}(X)$ is a functor $\omega: D_{\text {perf }}(X) \rightarrow D_{\text {perf }}(S)$ endowed with a bi-additive, bi-graded and bi-functorial isomorphism $\omega\left(M \otimes p^{*} E\right) \simeq \omega(M) \otimes E$, with $M \in D_{\text {perf }}(X), E \in$ $D_{\text {perf }}(S)$. An $S$-linear morphism between $S$-linear forms is defined as in $\$ 1.1$

We shall denote by $D_{\text {perf }}(X)^{*}$ the category of $S$-linear forms on $D_{\text {perf }}(X)$ and $S$ linear morphisms. One has a natural restriction functor $D(X / S)_{\text {perf }}^{*} \rightarrow D_{\text {perf }}(X)^{*}$.

By Proposition 1.10, if $K$ is an object of $D(X)$ of finite homological dimension over $S$, then $\omega_{K}: D(X) \rightarrow D(S)$ takes perfect complexes into perfect complexes. Then one has a functor

$$
D_{\text {fhd } / S}(X) \rightarrow D_{\text {perf }}(X)^{*} .
$$

Theorem 1.23. Assume that $X$ and $S$ are projective schemes over a field $k$. The functor

$$
\begin{aligned}
D_{\text {fhd } / S}(X) & \rightarrow D_{\text {perf }}(X)^{*} \\
K & \mapsto \omega_{K}
\end{aligned}
$$

is an equivalence.

Proof. The proof is similar to that of Theorem 1.14. Instead of Brown representability we need the following result: It is proved in [3] that any contravariant cohomological functor of finite type over $D_{\text {perf }}(X)$ ( $X$ a projective scheme over $k$ ) is representable by a bounded complex with coherent homology. Moreover, one has an equivalence between $D_{c}^{b}(X)$ and the category of contravariant cohomological functors of finite type over $D_{\text {perf }}(X)$ (see [1]). We shall refer to this result as the locally finite duality on $D_{\text {perf }}(X)$. 
It follows that if $\omega: D_{\text {perf }}(X) \rightarrow D_{\text {perf }}(S)$ is an $S$-linear form, it has a "right adjoint" $\omega^{\#}: D_{c}^{b}(S) \rightarrow D_{c}^{b}(X)$; that is, one has

$$
\operatorname{Hom}_{D(S)}(\omega(P), N) \simeq \operatorname{Hom}_{D(X)}\left(P, \omega^{\#}(N)\right)
$$

for any $P \in D_{\text {perf }}(X), N \in D_{c}^{b}(S)$. Now, the $S$-linearity of $\omega$ yields that $\omega^{\#}(E) \simeq$ $\omega^{\#}\left(\mathcal{O}_{S}\right) \otimes p^{*} E$ for any $E \in D_{\text {perf }}(S)$. Indeed, for any $P \in D_{\text {perf }}(X), E \in D_{\text {perf }}(S)$ one has

$$
\begin{aligned}
\operatorname{Hom}_{D(S)}\left(P, \omega^{\#}(E)\right) & \simeq \operatorname{Hom}_{D(X)}(\omega(P), E) \simeq \operatorname{Hom}_{D(X)}\left(\omega(P) \otimes E^{*}, \mathcal{O}_{S}\right) \\
& \simeq \operatorname{Hom}_{D(X)}\left(\omega\left(P \otimes p^{*} E^{*}\right), \mathcal{O}_{S}\right) \simeq \operatorname{Hom}_{D(S)}\left(P \otimes p^{*} E^{*}, \omega^{\#}\left(\mathcal{O}_{S}\right)\right) \\
& \simeq \operatorname{Hom}_{D(S)}\left(P, \omega^{\#}\left(\mathcal{O}_{S}\right) \otimes p^{*} E\right),
\end{aligned}
$$

where $E^{*}=\mathbf{R} \mathcal{H} \operatorname{Hom}_{S}^{\bullet}\left(E, \mathcal{O}_{S}\right)$. One concludes that $\omega^{\#}(E) \simeq \omega^{\#}\left(\mathcal{O}_{S}\right) \otimes p^{*} E$ by the locally finite duality on $D_{\text {perf }}(X)$.

Let us now see that $\omega^{\#}\left(\mathcal{O}_{S}\right)$ has finite homological dimension over $S$. Let us denote $L=\omega^{\#}\left(\mathcal{O}_{S}\right)$. For any $P \in D_{\text {perf }}(X), E \in D_{\text {perf }}(S)$ one has

$$
\begin{aligned}
\operatorname{Hom}_{D(S)}\left(E, \omega(P)^{*}\right) & \simeq \operatorname{Hom}_{D(S)}\left(E \otimes \omega(P), \mathcal{O}_{S}\right) \\
& \simeq \operatorname{Hom}_{D(S)}\left(\omega\left(p^{*} E \otimes P\right), \mathcal{O}_{S}\right) \\
& \simeq \operatorname{Hom}_{D(X)}\left(p^{*} E \otimes P, L\right) \\
& \simeq \operatorname{Hom}_{D(S)}\left(E, p_{*} \mathbf{R} \mathcal{H} m_{X}(P, L)\right) \\
& \simeq \operatorname{Hom}_{D(S)}\left(E, \omega_{L}\left(P^{*}\right)\right) .
\end{aligned}
$$

By the locally finite duality on $D_{\text {perf }}(X)$ one obtains $\omega(P)^{*} \simeq \omega_{L}\left(P^{*}\right)$. By Proposition 1.10 $L$ has finite homological dimension over $S$.

Let us denote $K=L^{\vee}$ and let us prove that $\omega \simeq \omega_{K}$. For any $P \in D_{\text {perf }}(X)$, $E \in D_{\text {perf }}(S)$ one has

$$
\begin{aligned}
\operatorname{Hom}_{D(S)}(\omega(P), E) & \simeq \operatorname{Hom}_{D(X)}\left(P, \omega^{\#}(E)\right) \simeq \operatorname{Hom}_{D(X)}\left(P, L \otimes p^{*} E\right) \\
& \simeq \operatorname{Hom}_{D(X)}\left(P, K^{\vee} \otimes p^{*} E\right) \simeq \operatorname{Hom}_{D(X)}\left(P, \mathbf{R} \mathcal{H o m} \dot{X}_{X}\left(K, p^{!} E\right)\right) \\
& \simeq \operatorname{Hom}_{D(S)}\left(p_{*}(P \otimes K), E\right)
\end{aligned}
$$

and then $\omega \simeq \omega_{K}$. The rest of the proof is as in Theorem 1.14.

Corollary 1.24. Under the same hypothesis, the natural restriction functor

$$
D(X / S)_{\text {perf }}^{*} \rightarrow D_{\text {perf }}(X)^{*}
$$

is an equivalence.

Now we shall study the linear dual of $D_{c}^{b}(X)$.

Definition 1.25. An $S$-linear form on $D_{c}^{b}(X)$ is a functor $\omega: D_{c}^{b}(X) \rightarrow D_{c}^{b}(S)$ endowed with a bi-additive, bi-graded and bi-functorial isomorphism

$$
\omega\left(M \otimes p^{*} E\right) \simeq \omega(M) \otimes E
$$

for any $M \in D_{c}^{b}(X), E \in D_{\text {perf }}(S)$.

Theorem 1.26. Assume that $X$ and $S$ are projective schemes over a field $k$. Then the functor

$$
\begin{aligned}
D_{\text {perf }}(X) & \rightarrow D_{c}^{b}(X)^{*} \\
K & \mapsto \omega_{K}
\end{aligned}
$$

is an equivalence. 
Proof. Let $\omega: D_{c}^{b}(X) \rightarrow D_{c}^{b}(S)$ be an $S$-linear form. It is proved in 8 that any locally-finite homological functor on $D_{c}^{b}(X)$ ( $X$ a projective scheme over a field $k$ ) is representable by an object of $D_{\text {perf }}(X)$. It follows (see [2, Prop. 2.2]) that the category of locally-finite homological functors on $D_{c}^{b}(X)$ is equivalent to $D_{\text {perf }}(X)$. Hence $\omega$ has a "left adjoint" $\widetilde{\omega}: D_{\text {perf }}(S) \rightarrow D_{\text {perf }}(X)$; that is, one has

$$
\operatorname{Hom}_{D(S)}(E, \omega(M)) \simeq \operatorname{Hom}_{D(X)}(\widetilde{\omega}(E), M)
$$

for any $E \in D_{\text {perf }}(S), M \in D_{c}^{b}(X)$. Now the $S$-linearity of $\omega$ yields that $\widetilde{\omega}(E) \simeq$ $\widetilde{\omega}\left(\mathcal{O}_{S}\right) \otimes p^{*} E$. Indeed,

$$
\begin{aligned}
\operatorname{Hom}_{D(X)}(\widetilde{\omega}(E), M) & \simeq \operatorname{Hom}_{D(S)}(E, \omega(M)) \simeq \operatorname{Hom}_{D(S)}\left(\mathcal{O}_{S}, E^{*} \otimes \omega(M)\right) \\
& \simeq \operatorname{Hom}_{D(S)}\left(\mathcal{O}_{S}, \omega\left(p^{*} E^{*} \otimes M\right)\right) \simeq \operatorname{Hom}_{D(X)}\left(\widetilde{\omega}\left(\mathcal{O}_{S}\right), p^{*} E^{*} \otimes M\right) \\
& \simeq \operatorname{Hom}_{D(X)}\left(\widetilde{\omega}\left(\mathcal{O}_{S}\right) \otimes p^{*} E, M\right) .
\end{aligned}
$$

Let us denote $K=\widetilde{\omega}\left(\mathcal{O}_{S}\right)^{*}$. Since $\widetilde{\omega}\left(\_\right) \simeq \widetilde{\omega}\left(\mathcal{O}_{S}\right) \otimes p^{*}(\ldots)$, one deduces, by "adjointness", that $\omega\left(\_\right) \simeq p_{*}\left(K \otimes \_\right)$. That is, $\omega \simeq \omega_{K}$.

Corollary 1.27. Assume also that $p: X \rightarrow S$ has finite Tor-dimension. The natural restriction functor

$$
D(X / S)_{b c}^{*} \rightarrow D_{c}^{b}(X)^{*}
$$

is an equivalence.

1.3. A linear criterion for integrality. Let $p: X \rightarrow S$ and $q: Y \rightarrow S$ be two $S$-schemes. For any base change $S^{\prime} \rightarrow S$ we denote $X_{S^{\prime}}=X \times{ }_{S} S^{\prime}$ and we still denote by $p: X_{S^{\prime}} \rightarrow S^{\prime}$ the morphism induced by $p: X \rightarrow S$ under base change.

Definition 1.28. Let $F: D(X) \rightarrow D(Y)$ be an $S$-linear functor and $f: S^{\prime} \rightarrow S$ a base change. We say that $F$ extends to $S^{\prime}$ if there exists an $S^{\prime}$-linear functor $F_{S^{\prime}}: D\left(X_{S^{\prime}}\right) \rightarrow D\left(Y_{S^{\prime}}\right)$ such that the diagram

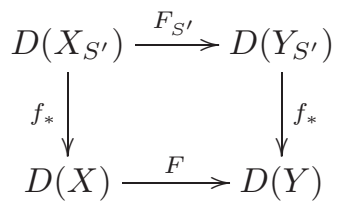

is commutative, i.e., $f_{*} \circ F_{S^{\prime}} \simeq F \circ f_{*}$.

Definition 1.29. An $S$-linear functor $F: D(X) \rightarrow D(Y)$ is said to be geometric if it extends to any base change $S^{\prime} \rightarrow S$.

Proposition 1.30. Assume that $q: Y \rightarrow S$ is flat. Let $F: D(X) \rightarrow D(Y)$ be an $S$-linear functor. Then $F$ is integral if and only if $F$ is geometric.

Proof. Assume that $F=\Phi_{K}$ is an integral functor of kernel $K \in D\left(X \times_{S} Y\right)$. Let $f: S^{\prime} \rightarrow S$ be a base change and $K_{S^{\prime}}=f^{*} K \in D\left(X_{S^{\prime}} \times_{S^{\prime}} Y_{S^{\prime}}\right)$. By the projection formula and flat base change it follows easily that

$$
\Phi_{K}\left(f_{*} M\right) \simeq f_{*} \Phi_{K_{S^{\prime}}}(M), \quad M \in D\left(X_{S^{\prime}}\right) .
$$

This means that $\Phi_{K}$ extends to $S^{\prime}$, an extension being $\Phi_{K_{S^{\prime}}}$.

Assume now that $F$ is geometric. Let us take $S^{\prime}=X, f=p$. By hypothesis there exists an $S^{\prime}$-linear functor $F_{S^{\prime}}: D\left(X \times_{S} S^{\prime}\right) \rightarrow D\left(Y \times_{S} S^{\prime}\right)$ such that $F\left(f_{*} M\right) \simeq$ $f_{*} F_{S^{\prime}}(M)$, for any $M \in D\left(X \times_{S} S^{\prime}\right)$. Let us denote by $\mathcal{O}_{\Delta} \in D\left(X \times_{S} S^{\prime}\right)$ the 
structure sheaf of the diagonal subscheme. For any $N \in D(X)$ one has $N \simeq$ $f_{*}\left(\mathcal{O}_{\Delta} \otimes p^{*} N\right)$. Then, by the $S^{\prime}$-linearity of $F_{S^{\prime}}$,

$$
F(N) \simeq F\left(f_{*}\left(\mathcal{O}_{\Delta} \otimes p^{*} N\right)\right) \simeq f_{*} F_{S^{\prime}}\left(\mathcal{O}_{\Delta} \otimes p^{*} N\right) \simeq f_{*}\left(F_{S^{\prime}}\left(\mathcal{O}_{\Delta}\right) \otimes q^{*} N\right) .
$$

This proves that $F$ is an integral functor of kernel $F_{S^{\prime}}\left(\mathcal{O}_{\Delta}\right)$.

\section{REFERENCES}

1. Matthew Robert Ballard, Derived categories of sheaves of quasi-projective schemes, Ph.D. thesis, Univ. of Washington, 2008. MR2712236

2. —_, Equivalences of derived categories of sheaves on quasi-projective schemes, arXiv:0905.3148v2 [math.AG].

3. Alexei Bondal and Michel Van den Bergh, Generators and representability of functors in commutative and noncommutative geometry, Mosc. Math. J. 3 (2003), no. 1, 1-36. MR1996800 (2004h:18009)

4. Daniel Hernández Ruipérez, Ana Cristina López Martín, and Fernando Sancho de Salas, Relative integral functors for singular fibrations and singular partners, Journal of the European Mathematical Society 11 (2009), 597-625. MR2505443(2010a:14029)

5. Amnon Neeman, The Grothendieck duality theorem via Bousfield's techniques and Brown representability, J. Amer. Math. Soc. 9 (1996), no. 1, 205-236. MR.1308405 (96c:18006)

6. D. O. Orlov, Equivalences of derived categories and K3 surfaces, Algebraic geometry, 7, J. Math. Sci. (New York) 84 (1997), no. 5, 1361-1381. MR.1465519 (99a:14054)

7. Dmitri D. Orlov and Valery A. Lunts, Uniqueness of enhancement for triangulated categories, J. Amer. Math. Soc. 23 (2010), 853-908.

8. Raphael Rouquier, Dimensions of triangulated categories, J. K-Theory 1(2) (2009), 193-256. MR2434186(2009i:18008)

Department of Mathematics, University of Salamanca, Plaza de la Merced 1-4, 37008 Salamanca, Spain

E-mail address: fsancho@usal.es

Department of Mathematics, University of Salamanca, Plaza de la Merced 1-4, 37008 Salamanca, Spain

E-mail address: mplu@usal.es 